\title{
Evaluation of the nonparametric estimation method in NONMEM VI: application to real data
}

UPPSALA UNIVERSITET
Paul G. Baverel, Radojka M. Savic, Justin J. Wilkins and Mats O. Karlsson Division of Pharmacokinetics \& Drug Therapy, Uppsala University, Sweden

\section{Background and Objective}

A nonparametric estimation method has been introduced to NONMEM in version VI. A previous study indicated that this new feature showed promising properties when analyzing simulated data, in that the nonparametric distribution of the parameter estimates closely matched the true distribution used in simulation [1]. However, experience with real data sets is thus far limited.

The aim of this study was to evaluate the predictive performance of the nonparametric estimation method in comparison with standard parametric methods when applied to real data sets

\section{Methods}

30 models developed using real data and a parametric method (FO or FOCE method in NONMEM) for 18 different drugs were used. Of these, 21 were pharmacokinetic in nature (one-, two-, and threecompartment models) and 9 pharmacodynamic (e.g. direct inhibitory $\mathrm{E}_{\max }$ models, indirect effect models).

The datasets used contained between 8 and 637 subjects, with between 2 and 45 observations per subject.

Four methods for estimating model parameters and parameter distributions were compared:

- FO method

- FOCE method

- NONP-FO method (nonparametric preceded by FO)

- NONP-FOCE method (nonparametric preceded by FOCE)

Numerical predictive checks (NPCs) were used to test the appropriateness of each model [2]. Up to 1000 new datasets were simulated from each model and with each estimation method and used to construct $95 \%$ and $50 \%$ prediction intervals (PIs). The percentages of total outliers (expected values being $5 \%$ and $50 \%$ for the $95 \%$ and $50 \%$ PIs, respectively) were obtained, as well as the percentages above and below the PIs, and the ratio of points above and below the median.

In order to estimate the predictive performance of each method, the mean absolute error (MAE, \%) and the mean error (ME, \%) were computed as indicators of imprecision and bias and compared using t-tests.

\section{Results and Discussion}

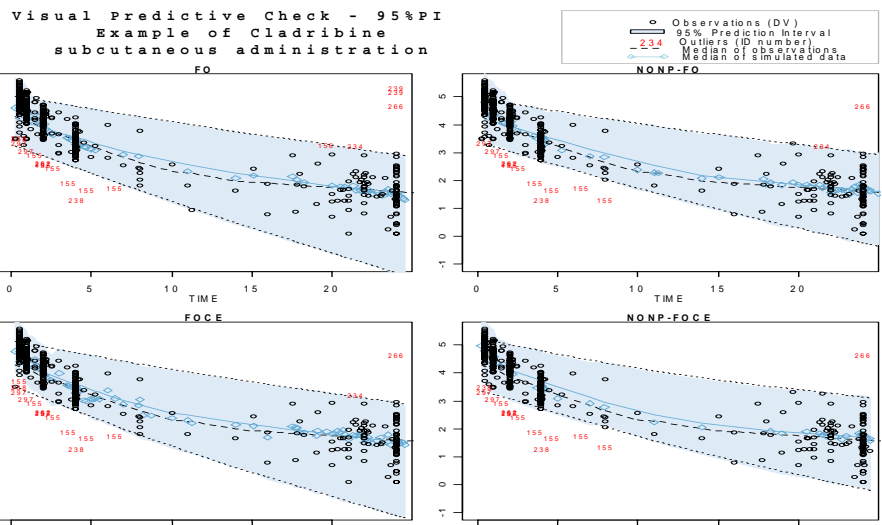

Figure 2. VPC plots of cladribine, representing the observed concentration (black circles) versus time and the $95 \%$ prediction interval (in light blue), when using 4 different estimation methods. On the left side, the parametric estimation methods (FO (top) and FOCE) have been used when running the adequate model and on the right side, the nonparametric estimation methods (NONP-FO (top) and NONP-FOCE) have been used.
Table 1 Measurements of imprecision (MAE, \%) for $95 \%$ and $50 \%$ Pls

The stars indicate the results of $t$-tests when the difference between measurements were statistically significant for a type-l error of $5 \%$

\begin{tabular}{cccc|ccc}
\hline M AE (\%) & FO & NONP-FO & P-value & FOCE & NONP-FOCE & P-value \\
\hline $95 \%$ U & 0.015 & 0.010 & $0.009 *$ & 0.013 & 0.009 & 0.067 \\
$95 \% \mathrm{~L}$ & 0.016 & 0.015 & 0.576 & 0.012 & 0.011 & 0.689 \\
Total 95\% & 0.018 & 0.017 & 0.754 & 0.016 & 0.015 & 0.426 \\
$50 \% \mathrm{U}$ & 0.051 & 0.027 & $0.001 *$ & 0.025 & 0.019 & 0.104 \\
$50 \% \mathrm{~L}$ & 0.047 & 0.033 & 0.059 & 0.041 & 0.031 & 0.019 * \\
Total 50\% & 0.060 & 0.050 & 0.080 & 0.051 & 0.038 & 0.007 * \\
Ratio median & 0.264 & 0.099 & $0.009 *$ & 0.168 & 0.118 & 0.012 *
\end{tabular}

Table 2 Measurements of bias (ME, \%) for $95 \%$ and $50 \%$ Pls:

\begin{tabular}{cccc|ccc}
\hline ME (\%) & FO & NONP-FO & P-value & FOCE & NONP-FOCE & P-value \\
\hline $95 \%$ U & 0.000 & -0.001 & 0.592 & 0.000 & -0.001 & 0.637 \\
$95 \% \mathrm{~L}$ & 0.007 & 0.001 & $0.015 *$ & 0.005 & 0.002 & 0.124 \\
Total 95\% & 0.008 & 0.000 & $0.01 *$ & 0.005 & 0.001 & 0.088 \\
$50 \% \mathrm{U}$ & 0.023 & 0.008 & 0.129 & 0.002 & 0.001 & 0.727 \\
$50 \% \mathrm{~L}$ & 0.018 & 0.020 & 0.809 & 0.024 & 0.016 & 0.092 \\
Total 50\% & 0.041 & 0.029 & 0.054 & 0.026 & 0.016 & 0.081 \\
Ratio median & -0.025 & -0.025 & 1.000 & -0.060 & -0.051 & 0.733 \\
\hline
\end{tabular}

- There was a tendency to simulate more variability than was observed with both FO alone and FOCE alone, but this was not the case with NONP-FO and NONP-FOCE

- Overall, less imprecision and less bias were observed with nonparametric methods than with parametric methods (FO and FOCE)

- T-tests revealed that imprecision related to the ratio of points above / below the median was significantly lower $(p<0.05)$ with nonparametric methods than with parametric methods

- Regarding the percentages of total outliers for the PIs concerned: - Imprecision was significantly lower for NONP-FOCE than FOCE at $50 \% \mathrm{PI}$, but not at $95 \% \mathrm{PI}$

- Bias was significantly lower for NONP-FO than FO at 95\%PI - Imprecision was significantly lower for NONP-FO than FO at $95 \%$ and $50 \%$ PIs of outliers above

These models had been developed and accepted previously as final using parametric methods. Some showed good simulation properties from the start and thus could not be expected to be improved upon by nonparametric methods.

Moreover, the shrinkage related to empirical Bayes estimates (EBEs) has not been taken into account [3]. It may have affected performance since the EBEs are taken as the support points of the nonparametric distribution.

In spite of these 2 points, nonparametric methods showed simulation properties that were better overall than parametric for a wide range of different PK and PD models.

\section{Conclusion}

When applied to real datasets and evaluated by predictive checks, the nonparametric estimation methods in NONMEM VI performed better than the corresponding parametric methods (FO or FOCE) with less imprecision and less bias for the majority of the outcomes investigated in this study.

References:

1. Savic RM, Kjellsson M, Karlsson MO. Evaluation of the nonparametric estimation method in NONMEM VI beta PAGE 15 (2006) Abstract 937

2. Wilkins JJ, Karlsson MO, Jonsson EN. Patterns and power for the visual predictive check. PAGE 15 (2006) Abstract 1029

3. Savic RM, Wilkins J], Karlsson MO. (Un)informativeness of Empirical Bayes Estimate Based Diagnostics. AAPS, San Antonio, 2006 